\title{
Expression and functional analysis of apoptosis-related gene BmICAD in silkworm, Bombyx mori
}

\author{
Wei Yu*, Yue Ma and Yao-Zhou Zhang \\ Institute of Biochemistry, College of Life Sciences, Zhejiang Sci-Tech University, Hangzhou 310018, China. \\ Accepted 27 July, 2011
}

\begin{abstract}
The ICAD gene plays an important role in the growth and development processes in insects. We conducted a molecular cloning and functional analysis to study a specific silkworm gene BmICAD related to apoptosis. The BmICAD gene was obtained from the fifth instar larvae of the silkworm by RTPCR and over-expressed in Escherichia coli as His-tagged fusion proteins. Subcellular localization of the protein indicated that BmICAD was found in the cytoplasm near the nucleus. RNAi assay indicated that the apoptosis rate of Bm5 cells increased markedly. A His pull-down assay was used to investigate proteins that bind to rBmICAD. Two proteins, DNA supercoiling factor (SCF) and secreted protein acidic and rich in cysteine (SPARC), were found. These results indicate that down regulation of BmICAD can increase the apoptosis rate of Bm5 cells, and that SCF and SPARC may have important roles by interacting with BmICAD during this process.
\end{abstract}

Key words: Bombyx mori, BmICAD, subcellular localization, RNAi, protein interaction.

\section{INTRODUCTION}

Apoptosis, or programmed cell death (PCD), is an essential process during normal development, cell senescence, cell damage and homeostasis of a multicellular organism (Van de Craen et al., 1997; Feng et al., 2004). Apoptosis is the process of cellular selfdestruction, regulated by increasing the number of genes that are conserved in all metazoans and recognized by characteristic morphological and molecular changes.

DNA fragmentation is a prominent feature of apoptosis (Wyllie, 1980). DFF (DNA fragmentation factor)-caused DNA breaks is an important step in apoptosis in vertebrate cells (Eckhart et al., 2007). DFF is a protein complex, composed of the inhibitor of caspase-3activated DNAase (ICAD) and caspase-activated DNase (CAD) (Enari et al., 1998; Liu et al., 1997). ICAD cannot

*Corresponding author. E-mail: yuwei@zstu.edu.cn. Tel: +86 (0)571 86843190. Fax: +86 (0)57186843191.

Abbreviations: OFR, Open reading frame; CIDE, cell deathinducing DFF45-1ike effectors; CAD, caspase-activated Dnase; ICAD, inhibitor of caspase-3-activated DNAase; DFF, DNA fragmentation factor; PCD, programmed cell death; SCF, supercoiling factor; SPARC, secreted protein acidic and rich in cysteine. only inhibit the activity of CAD DNase, but also has a chaperone-like activity specific for CAD (Sakahira et al., 1998). Caspase 3 is a protease that is activated during apoptosis and can cleave and inactivate its CADinhibitory effect, releasing CAD to enter the nucleus and thus cause DNA cleavage and nuclear enrichment (Sakahira et al., 1998; Liu et al., 1998). Previous studies showed that both DFF45/ICAD and DFF40/CAD contain a motif known as a CIDE (cell death-inducing DFF45-1ike effectors) domain. It has been shown previously that the homologous N-terminal domains of CAD and ICAD bind to each other and that this binding is a crucial step in formation of the complex between CAD and ICAD (Liu et al., 1998). CIDEs are pro-apoptotic proteins that induce DNA fragmentation as well as other apoptotic features such as membrane blebbing and nuclear condensation (Hoffmann et al., 2004; Inohara et al., 1998).

Bombyx mori is an important model organism for Lepidoptera, undergoing complete metamorphosis (Kuroda et al., 1989). PCD is crucial in body restructuring during metamorphosis of holometabolous insects (Tettamanti et al., 2007); it is therefore important to study the mechanism of apoptosis during the metamorphosis of silkworm. Most studies on ICAD have been limited to gene cloning, sequence analysis and expression (Wang et al., 2006); there are few reports on the apoptotic 
mechanisms of ICAD. In this study, we identified a silkworm ICAD complementary DNA (cDNA; Bm-ICAD) from the fifth instar larvae of Bombyx mori by RT-PCR. Using bioinformatic analysis, the open reading frame (ORF) of BmICAD gene, molecular weight and conserved domain of the BmICAD protein were predicted. Immunocytochemistry and Western-blot analysis were used to determine the subcellular localization and expression levels of BmICAD in E. coli. Furthermore, the biological function of BmICAD was studied by RNA interference analysis and possible interaction proteins with BmICAD were identified by pull-down assays. The results provide an important foundation for further studies on the functional roles of BmICAD during the metamorphosis of silkworm.

\section{MATERIALS AND METHODS}

\section{Bioinformatic analyses}

The BmICAD gene was obtained from the fifth instar larvae of Bombyx mori by RT-PCR. The similarity analysis of nucleotide and protein sequences was performed using the BLAST algorithm from NCBI (http://www.ncbi.nlm.nih.gov/BLAST/). The characteristics of this gene were analyzed using DNAstar software.

\section{Insects, strains, growth media and reagents}

The Bombyx mori strain used in this study was the progeny of Jingsong $\times$ Haoyue. Silkworms were reared on mulberry leaves under standard conditions. The fifth instar larvae were dissected, frozen immediately in liquid nitrogen, and stored at $-80^{\circ} \mathrm{C}$. Escherichia coli strains TG1 and BL21 (DE3) were grown at $37^{\circ} \mathrm{C}$ in LB media, $\mathrm{pH} 7.5$, containing $5 \mathrm{~g}$ yeast extract, $10 \mathrm{~g}$ tryptone peptone, and $10 \mathrm{~g} \mathrm{NaCl}$ per liter. Recombinant $E$. coli strains were grown in the presence of kanamycin $(50 \mu \mathrm{g} / \mathrm{ml})$. Bm5 cells (a gift from Prof. Zhang ZF) were cultured in TC-100 medium (Sigma) supplemented with $10 \%$ fetal calf serum (FCS, Gibco BRL) at $27^{\circ} \mathrm{C}$. 4'-6-Diamidino-2-phenylindole (DAPI) was purchased from Sigma. Cy3-labeled goat anti-rabbit IgG was purchased from Proteintech Group Inc.

\section{Cloning, expression, purification and antibody preparation}

Total RNA was extracted from silkworm fifth instar larvae using Trizol regent (Invitrogen) as instructed by the manufacturer. The Ready-To-Go T-Primed First-Strand Kit (Amersham) was used to synthesize first-strand cDNAs from total RNA. Using the first-strand cDNA as a template, the BmICAD ORF was amplified by polymerase chain reaction (PCR). Based on the BmICAD cDNA sequence, a pair of primers was designed. For subcloning purposes, two restriction endonuclease recognition sites, Sacl and $X$ hol, were included at the 5 '-ends of the primers. The forward primer was P1 5'-CGAGCTCATGGAAAACGACATTAAC-3', and the reverse primer was P2 5'- CCCTCGAGTTAGCAATTCCATAGC-3'. The $B m I C A D$ ORF was subcloned into the prokaryotic expression vector pET-28a $(+)$ to construct the recombinant plasmid $\mathrm{pET}$ 28a $(+)-B m I C A D$. Escherichia coli BL21 (DE3) cells were transformed with the recombinant plasmid. The transformed $E$. coli were grown in culture, and recombinant protein expression was induced by incubation at $25^{\circ} \mathrm{C}$ for $12 \mathrm{~h}$ with $1 \mathrm{mM}$ IPTG. The recombinant His-tagged fusion protein was extracted from the bacteria and purified using Ni-Sephadex ${ }^{\mathrm{TM}}$ G-25 Superfine (HiTrap $^{\text {TM }}$ Desalting Column, Amersham) metal-chelating affinity chromatography. Purified $6 \times \mathrm{His}-\mathrm{BmICAD}$ was used to generate anti-BmICAD polyclonal antibody in a New Zealand rabbit. Antiserum from the immunized rabbit was passed through a Protein A HP chromatography column to obtain purified rabbit IgG. ELISA was used to determine the polyclonal antibody titer, and the specificity of the polyclonal antibody was determined by Western blot analysis; the purified polyclonal antibody was diluted 1:1,000 and incubated with the immunoblot at room temperature for $2 \mathrm{~h}$, the goat anti-rabbit secondary antibody was diluted 1:1,000.

\section{Subcellular localization of BmICAD}

Bm5 cells were cultured overnight on glass cover slips and grown to $50-60 \%$ confluence, washed for 10 min three times in phosphate buffered saline (PBS), and fixed (3.7\% poly-formaldehyde) at room temperature for $15 \mathrm{~min}$. The cells were then permeabilized by incubation with $0.2 \%$ Triton X-100 at room temperature for $5 \mathrm{~min}$ and washed three times in PBS. The cells were blocked in $5 \%$ BSA in PBS at room temperature for $2 \mathrm{~h}$, followed by three $10 \mathrm{~min}$ washes in PBS containing 0.05\% Tween-20 (PBST). Cells were then incubated with antiserum containing anti-BmICAD polyclonal antibody (diluted 1:200 in blocking buffer) at $4^{\circ} \mathrm{C}$ overnight. Simultaneously, control cells were incubated with the negative serum obtained from the rabbit before immunizing with the antigen. After three 10-min washes in PBST, cells were incubated with Cy3labeled goat anti-rabbit IgG (diluted 1:1500 in PBS; Promega) at $37^{\circ} \mathrm{C}$ for $2 \mathrm{~h}$ and then washed twice for $10 \mathrm{~min}$ in PBST. Cells were then incubated with 4'-6-diamidino-2-phenylindole (diluted 1:2000 in PBS; Promega) at room temperature for $15 \mathrm{~min}$, and washed three times in PBST. Cells were examined using a Nikon ECLIPSE TE2000-E Confocal Microscope; images were analyzed using EZC1software.

\section{RNAi and cell transfection}

To determine the biological function of BmICAD, Bm5 cells were transfected with $B m I C A D$ dsRNA. The BmICAD ORF was amplified by PCR using primers (Pa, GGATCCTAATACGACTCACTATAG GATGGAAAACGACATTAAC, Pb, GGATCCTAATACGACT CACTATAGGTTAGCAATTCCATAGC $\mathrm{P} 1$ and P2) containing T7 promoter sequences (underlined). pET-28a ${ }^{(+)}-\mathrm{BmICAD}$ was used as a template for the PCR, and the two single strands were amplified using two primer pairs: $\mathrm{Pa}$ and $\mathrm{P} 2$, and $\mathrm{Pb}$ and $\mathrm{P} 1$. Using the $\mathrm{T} 7$ RiboMAXExpress RNAi System kit (Promega), the dsRNA was synthesized and the PCR products were purified according to the manufacturer's instructions. The products were quantified spectrophotometrically at $260 \mathrm{~nm}$ and $280 \mathrm{~nm}$, and sizes were confirmed by agarose gel electrophoresis. Silkworm Bm5 cells were seeded in 24-well plates containing complete TC-100 medium and left overnight to attach, then the cells were transfected with $B m I C A D$ dsRNA (0.9 $\mu \mathrm{g} / \mathrm{well})$ using liposomes (Invitrogen, Carlsbad) in serum-free media. Cells transfected with liposome was using as negative control. Following continuous culture for 6 hours, media was with fresh normal growth media and incubated for a further $72 \mathrm{~h}$ at $27^{\circ} \mathrm{C}$.

\section{Detection of BmICAD protein expression by Western blot}

Both BmICAD dsRNA transfected cells and control group cells were harvested and lysed in PBS. The lysates were centrifuged at 12000 $\mathrm{r} / \mathrm{min}$ at $4^{\circ} \mathrm{C}$ for $20 \mathrm{~min}$ to collect the supernatants. The proteins concentration was determined by Bradford methods. For each 


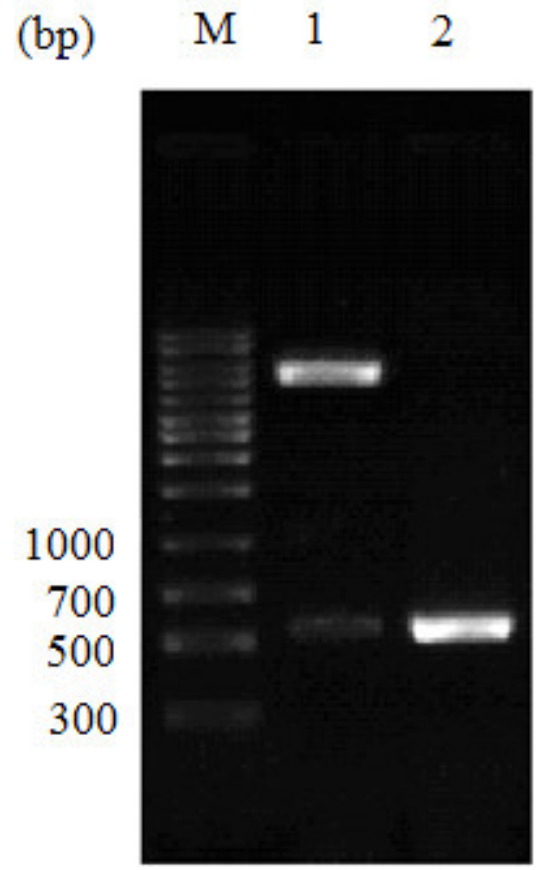

Figure 1. The identification of recombinant plasmid pET28a(+)$B m I C A D$. M, DNA ladder marker; lane 1, recombinant plasmid digested with Sacl/Xhol; Lane 2, the amplification products of PCR using pET28a (+)$B m I C A D$ as a template.

protein sample, $20 \mu \mathrm{g}$ proteins were loaded to each well of a $10 \%$ SDS-PAGE gel and electrotransferred onto PVDF membrane. After blocking with $5 \%$ non-fat milk for $1 \mathrm{~h}$ at room temperature, the membranes were incubated with the purified primary polyclonal antibody diluted by $1: 1,000$ at room temperature for $2 \mathrm{~h}$ at $37^{\circ} \mathrm{C}$. After three washes with TBST for 15 min each at room temperature, the membranes were incubated with the goat anti-rabbit secondary antibody diluted by 1:1,000. Densitometric analysis was performed using an image scanner and analyzing software $(\mathrm{NIH}$ image Ver. 1.61).

\section{Evaluation of $\mathrm{Bm} 5$ cell viability}

Bm5 cells were seeded in 96-well plates containing complete TC100 media and left overnight to attach. The complete media was then removed and the cells were treated with dsRNA for $72 \mathrm{~h}$ or with a UV crosslinker (model UVC2500) for $10 \mathrm{~min}$. After $10 \mathrm{~min}$ UV radiation treatment, cells were cultured in complete media for another $72 \mathrm{~h}$. The Bm5 cells viability was estimated after treatment with dsRNA, UV radiation, or dsRNA plus UV radiation; cells transfected with liposomes were used as a negative control and those treated with UV radiation as a positive control. The effectiveness of the RNAi or UV radiation experiment for $72 \mathrm{~h}$ was determined using the MTT (3-(4, 5-dimethylthiazol-2-yl) -2, 5diphenyltetrazolium bromide; Sigma) assay. Briefly, $1 \mathrm{mg} / \mathrm{ml}$ MTT (final concentration) was added to the well and incubated for $4 \mathrm{~h}$ at $27^{\circ} \mathrm{C}$. The medium was removed and cells were lysed with $2-$ isopropanol containing $0.04 \mathrm{~mol} / \mathrm{l} \mathrm{HCl}$. The absorbance measured at $490 \mathrm{~nm}$ was used to calculate the relative cell viability ratio (Dimmeler et al., 1997).

\section{Evaluation of $\mathrm{Bm} 5$ cells apoptosis}

The effects of RNAi on cell growth and death were determined by examining chromosomal DNA fragmentation. For quantitative determination, Bm5 cell apoptosis was measured as DNA fragmentation. DNA fragmentation was evaluated by measuring histone-associated DNA fragments using a photometric enzyme immunoassay (Cell Death Detection ELISA, Roche), according to the manufacturer's instructions. Briefly, attached cells were harvested and the cell suspension was pelleted by centrifugation. Floating and attached cells were lysed. After centrifugation, the supernatant was measured by ELISA (Yu et al., 2006).

\section{In vitro binding assays}

An in vitro His pull-down assay was used to identify the interactions between BmICAD and total extracted protein from Bm5 cells. The $\mathrm{Bm} 5$ cells were collected and lysed in PBS, the total protein were extracted for binding assays. The total protein $(10 \mu \mathrm{g})$ and purified 6xHis-BmICAD $(10 \mu \mathrm{g})$ were loaded onto a $0.1 \mathrm{ml} \mathrm{HiTrap}^{\mathrm{TM}}$ desalting column (Amersham) overnight at $4{ }^{\circ} \mathrm{C}$. The mixture was transferred to a $2 \mathrm{ml}$ tube and washed three times with $1.5 \mathrm{ml}$ of wash buffer $(20 \mathrm{mM}$ Tris $-\mathrm{HCl}(\mathrm{pH} 7.4), 0.5 \mathrm{M} \mathrm{NaCl}$ and $20 \mathrm{mM}$ imidazole), centrifuged for $10 \mathrm{~s}$ at $12000 \mathrm{~g}$ at $4^{\circ} \mathrm{C}$. The pellet was washed three times with $1.5 \mathrm{ml}$ of elution buffer $(20 \mathrm{mM}$ Tris- $\mathrm{HCl}$ (pH 7.4), $0.5 \mathrm{M} \mathrm{NaCl}$ and $200 \mathrm{mM}$ imidazole), centrifuged for $10 \mathrm{~s}$ at $12000 \mathrm{~g}$ at $4^{\circ} \mathrm{C}$. Samples without purified $6 \times \mathrm{His}-\mathrm{BmICAD}$ or $\mathrm{Bm} 5$ cell total protein were used as negative controls. Samples were separated by SDS-PAGE and digested with trypsin; peptide sequences were determined using an ABI4700MALDI-TOF /TOF MS. The obtained peptide mass fingerprinting (PMF) was searched in the NCBI protein data bank.

\section{RESULTS}

\section{Bioinformatic analyses}

The BmICAD cDNA was 844 bp in length and contained a 522 bp ORF (269-791 bp) which encoding a protein of 174 amino acids with a predicated molecular weight of $19.6 \mathrm{kDa}$. Using NCBI BLAST, we found that this protein contains an $\mathrm{N}$-terminal CIDE-N domain that was conserved in other known pro-apoptotic CIDE-N proteins, a novel family of cell death activators. These results reveal that BmICAD is a member of this family and its biological activity is possibly similar to the ICAD in mammals. In normal cells it might also act as the inhibitor of CAD by its binding to CAD.

\section{Prokaryotic expression of BmICAD and preparation of polyclonal antibody}

The BmICAD ORF was subcloned into the pET-28a (+) prokaryotic expression vector (Figure 1). A BmICAD fusion protein was expressed successfully in E. coli. The molecular weight of the recombinant fusion protein, as determined by electrophoresis, was $25 \mathrm{kDa}$, which is consistent with the predicted value (Figure $2 \mathrm{~A}$ ). After $\mathrm{Ni}^{2+}$ affinity chromatography, recombinant BmICAD was highly purified as detected by electrophoresis (Figure2B). 
A.

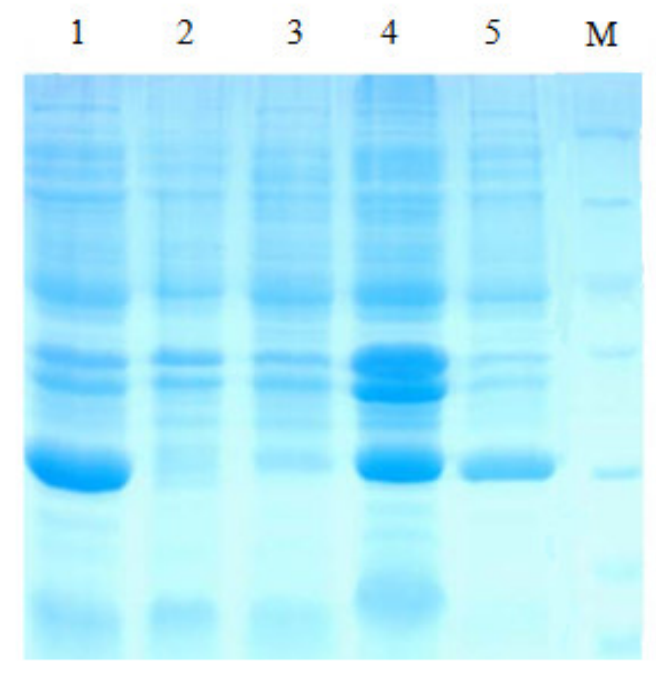

$(\mathrm{kD})$

116

66.4

45

35

25

18.4
B.

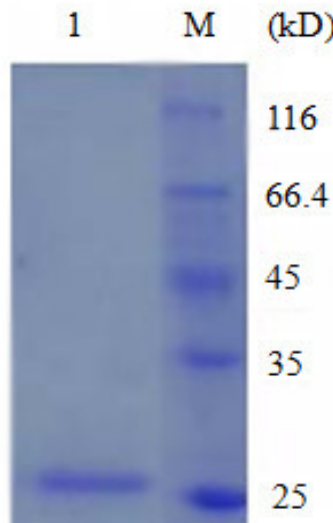

18.4

Figure 2. Expression and purification of $r B m I C A D$. A. lane $M$, protein molecular-weight marker (values are in $\mathrm{kDa}$ ); lane 1, total extract of BL21-pET-28a-BmICAD bacteria after induction with IPTG; lane 2, total extract of BL21-pET-28a(+) bacteria after induction with IPTG; lane 3, total extract of BL21-pET-28a-BmICD bacteria without induction; lane 4, the precipitate of sonicated lysate; lane 5 , the supernatant of sonicated lysate. B. lane M, protein molecular-weight marker (values are in $\mathrm{kDa}$ ); lane 1, purified His-tagged BmICAD.

The anti-BmICAD polyclonal antibody titer was determined by ELISA, positive serum extinction value/negative serum extinction value $(P / N) \geq 2.1$ was positive; $1.5 \leq \mathrm{P} / \mathrm{N}<2.1$ was suspicious expression; $\mathrm{P} / \mathrm{N}$ $<1.5$ was negative. Finally, the titer of the antibodies was determined to be $1: 25,600$, which could meet the requirements for all next experiments. Western blot was used to analyze the specificity of BmICAD polyclonal antibodies; the antibody was incubated with the prokaryotic expression product. The results indicate that the antibody could react specifically with rBmICAD.

\section{Subcellular Localization of BmICAD}

The cells that had been treated with Cy3-labeled goat anti-rabbit IgG and DAPI were examined using a Nikon ECLIPSE TE2000-E Confocal Microscope, and images were analyzed using EZ-C1software. Cy3-labeled goat anti-rabbit IgG emits red fluorescence when stimulated with light of wavelength $550 \mathrm{~nm}$, and DAPI-stained nuclei emit blue fluorescence when stimulated with light of wavelength $353 \mathrm{~nm}$. Our results reveal that BmICAD is mainly located in the cytoplasm near the nucleus (Figure 3).

\section{Effect of BmICAD dsRNA transfection on BmICAD expression}

To test the role of BmICAD in the process of $\mathrm{Bm} 5$ cell apoptosis, dsRNA against BmICAD were synthesized. $1 \%$ agarose gel electrophoresis showed that the size of synthetic dsRNA was consistent with the predicted value. The absorbance of a diluted dsRNA (40x) sample was measured at 260 and $280 \mathrm{~nm}$; the A260/A280 ratio was between 1.8 and 2.0 and the concentration of the BmICAD dsRNA was $1.87 \mu \mathrm{g} / \mu \mathrm{l}$. Synthesized dsRNA was transfected into the Bm5 cell, normal cells transfected with liposome only were used as a negative control. Western blot demonstrated that synthesized dsRNA could inhibit the expression of BmICAD protein effectively (Figure 4) and it was therefore used for further studies.

\section{Effect of RNAi on Bm5 cell viability}

To determine the role of BmICAD in Bm5 cell viability, MTT assay was used to test the cell viability after RNAi and UV radiation treatment. MTT experiments demonstrated that the optimal concentration of the BmICAD dsRNA for use in RNAi experiments was $0.9 \mu \mathrm{g} / \mathrm{well}$ and that the optimal duration of the interference was $72 \mathrm{~h}$ (data not shown). The MTT results showed that the activity of $\mathrm{Bm} 5$ cells decreased significantly following RNAi or UV radiation treatment, indicating that silencing the BmICAD gene resulted in increased cell death (Figure 5).

\section{Effect of RNAi on Bm5 cell apoptosis}

To determine whether BmICAD gene plays an important 

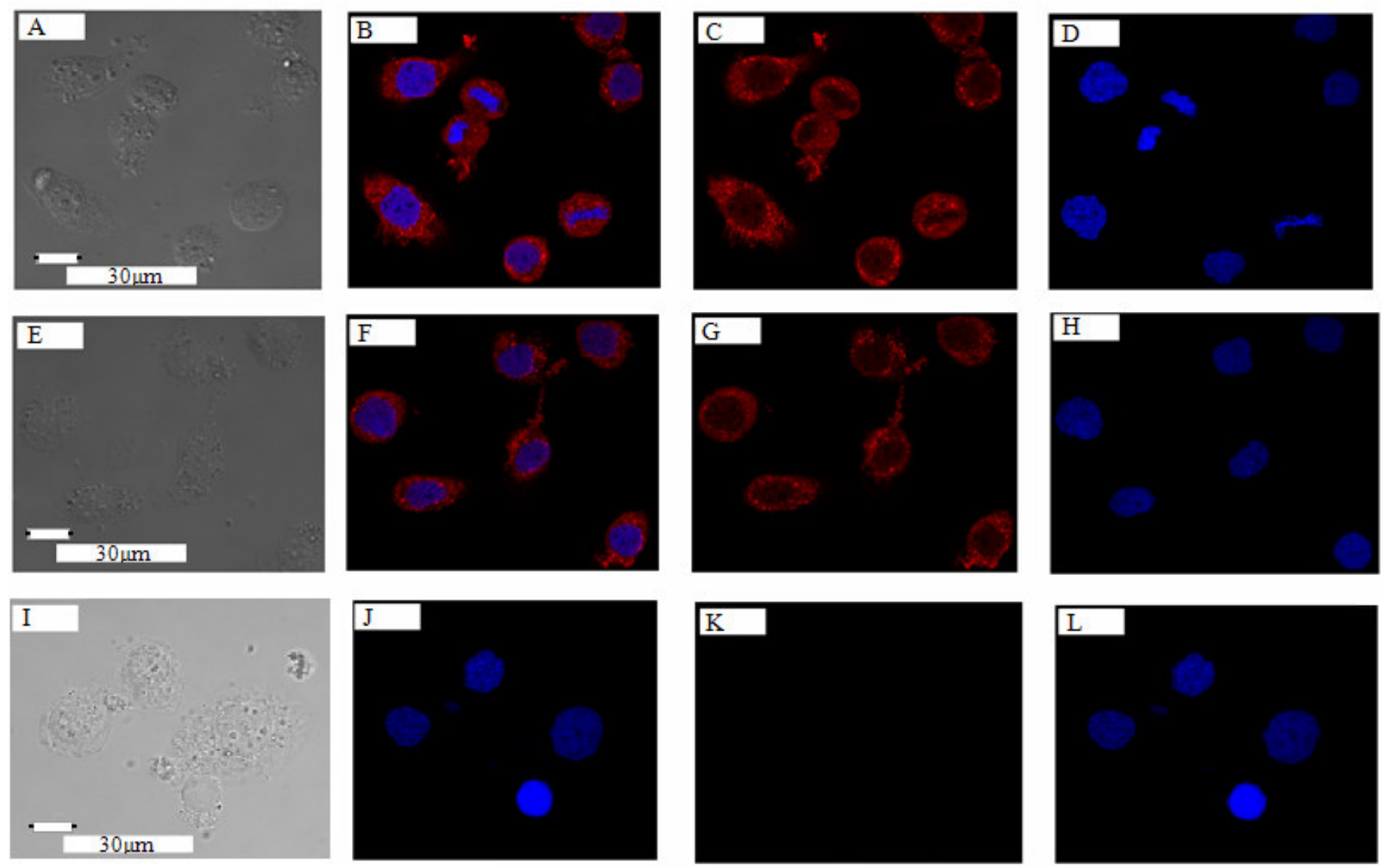

Figure 3. The subcellular localization of BmICAD. A, E, I. Cells in the light transmission. B, F, J. Merged image. C, G, K. Intracellular BmICAD stained with Cy3. D, H, L. Nucleolus stained with DAPI. I-L.Negative control. Scale bars represent $30 \mu \mathrm{m}$.

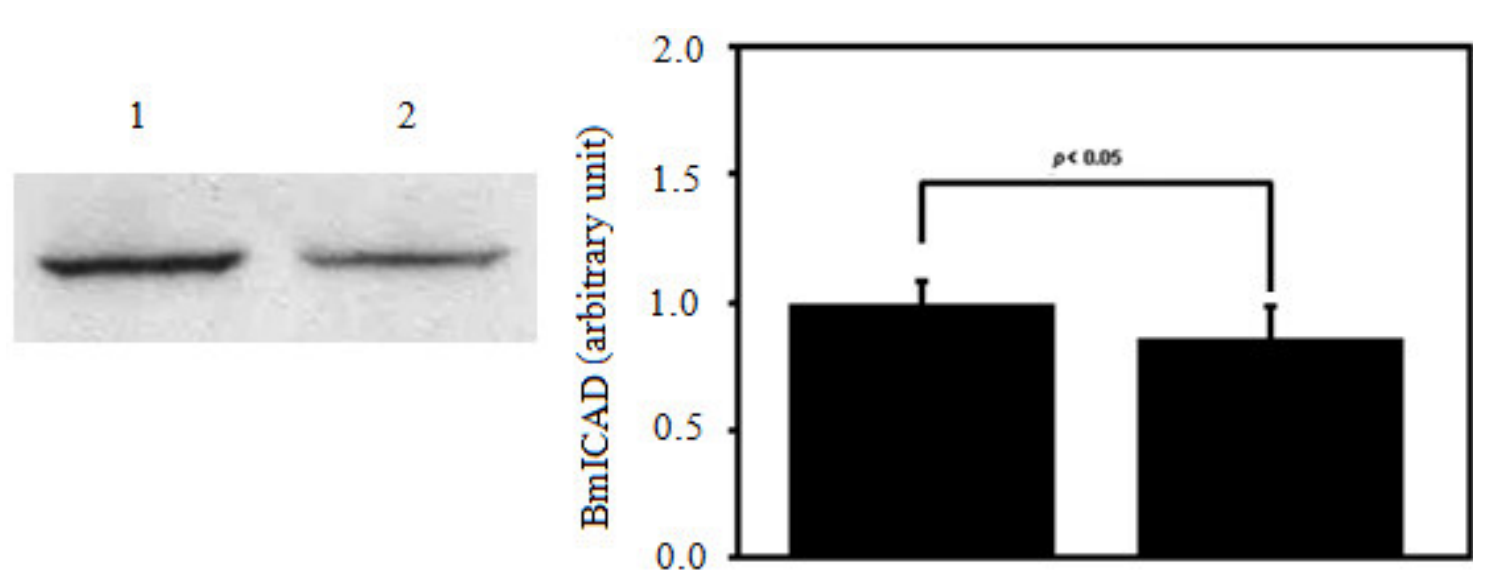

Figure 4. Western blot analysis of $B m I C A D$ dsRNA interference. Lane 1, control group: cells were transfected with liposome; lane 2, RNAi group: cells were transfected with BmICAD dsRNA. Right panels show the results of densitometric analyses of immunoblotting (mean \pm SEM, $n=3$ ).

role in the process of Bm5 cells apoptosis, RNAi and UV radiation treatment were carried out to test the rate of cells apoptosis. As measured by DNA fragmentation,
Bm5 cells apoptosis was significantly increased by RNAi or UV radiation treatment and was particularly increased after treatment with dsRNA and UV radiation, compared 


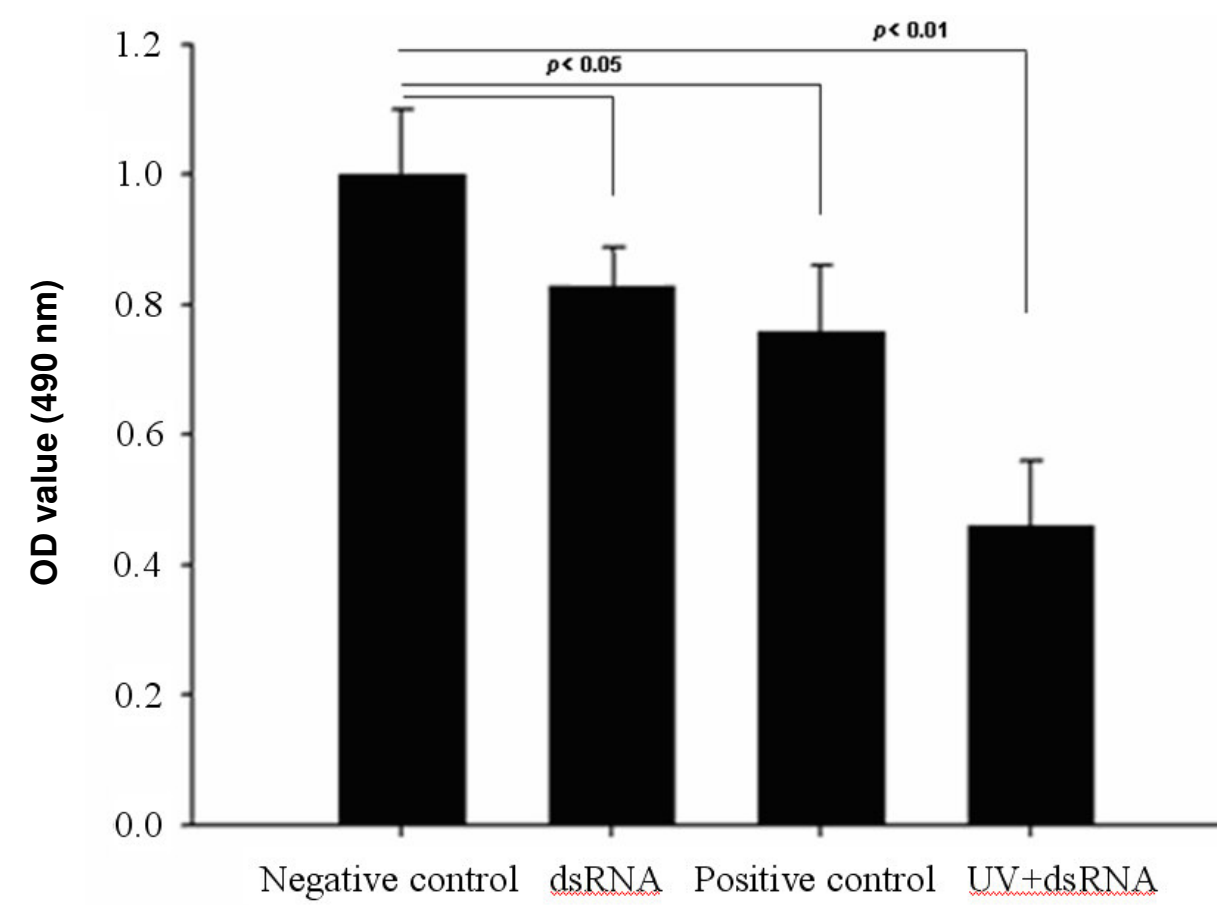

Figure 5. Effect of RNAi or UV radiation treatment on $\mathrm{Bm} 5$ cell viability. Negative control, cells were transfected with liposome; dsRNA, cells were treated with BmICAD dsRNA for $72 \mathrm{~h}$; positive control, cells were treated with UV radiation for $10 \mathrm{~min}$; UV+dsRNA, cells were treated with UV radiation for 10 min and BmICAD dsRNA for $72 \mathrm{~h}$. Cell viability was evaluated at $72 \mathrm{~h}$ after dsRNA treatment or UV radiation by measuring the optical density at $490 \mathrm{~nm}$ (mean \pm SEM, $\mathrm{n}=3$ ).

with the control group (Figure 6). Consistent with this effect, changes in cell viability was also decreased by RNAi or UV radiation treatment (Figure 5).

\section{Analysis of BmICAD-binding proteins}

To analyze proteins that interact with BmICAD, samples eluted from HiTrap ${ }^{\mathrm{TM}}$ Desalting columns were separated by SDS-PAGE (Figure 7). Compared with the negative control groups (without purified 6xHis-BmICAD), five different protein points were selected and peptide sequences were further determined by ABI4700MALDITOF/TOF MS after trypsin digests. After screening the protein sequence databases, two possible interacting proteins, BmSCF (DNA supercoiling factor) and BmSPARC (secreted protein acidic and rich in cysteine), were identified (Table 1).

\section{DISCUSSION}

Apoptosis has a very important role during larval tissue destruction in the silkworm. Previous studies revealed that ICAD is involved in this process because it is the substrate of activated caspase 3 during the execution phase of apoptosis (Yokoyama et al., 2000). However, it was unknown whether BmICAD is also involved in the execution of cell death as ICAD in other species and its role during the process of cell death. In this study, we cloned and expressed the BmICAD gene in E. coli and analyzed the possible relationship between BmICAD and apoptosis by RNAi and DNA fragmentation. The results show that silencing the BmICAD gene resulted in increased cell death and downregulation of cell activity. These results are in agreement with previous results in HeLa cells (Tsuruta et al., 2007). They suggested that RNAi could decrease the level of CAD and further inhibited internucleosomal DNA degradation in HeLa cell. In normal cells, CAD is produced as a complex with ICAD; ICAD specifically inhibits CAD-induced degradation of nuclear DNA and DNase activity (Enari et al., 1998; Liu et al., 1997). So the effect of CAD RNAi and the binding of CAD/ICAD complex are similar, both of them can decrease cell apoptosis by downregulating the activity of CAD. Our results suggest that BmICAD dsRNA might promote cell apoptosis by downregulating the expression of BmICAD and therefore releases CAD, allowing $C A D$ to enter the nucleus and degrade chromosomal DNA.

Immunostaining of endogenous ICAD showed it to be in the nucleus of human epithelial and HeLa cells (Liu et 


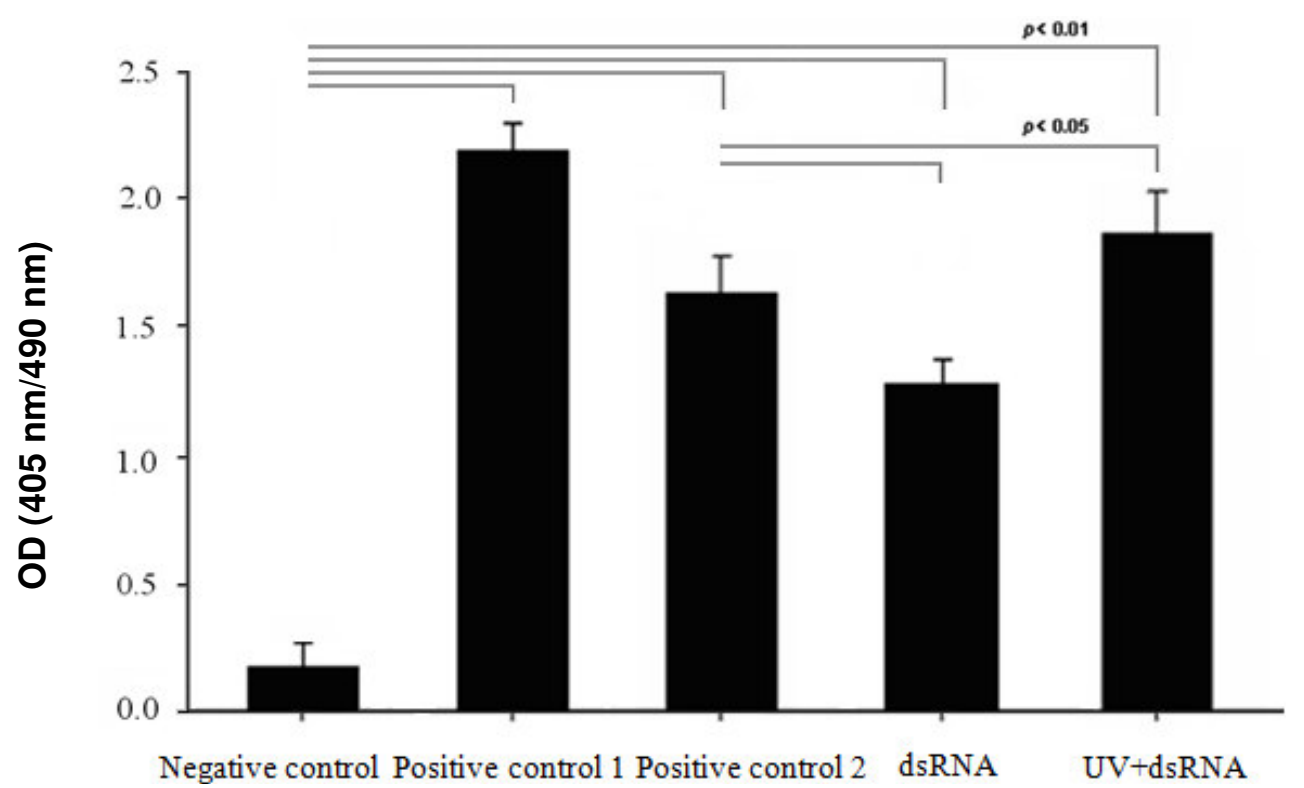

Figure 6. Detecting the effect of BmICAD RNAi or UV radiation treatment on Bm5 cell death by ELISA. Negative control, cells were transfected with liposome; positive control 1, DNA-histone complex (provided with kit); positive control 2, cells were treated with UV radiation for 10 min; dsRNA, cells were treated with dsRNA of $B m I C A D$ for $72 \mathrm{~h}$; UV+dsRNA, cells were treated with UV radiation for $10 \mathrm{~min}$ and dsRNA of $B m I C A D$ for $72 \mathrm{~h}$. Cell apoptosis was evaluated by measuring the optical density at $405 \mathrm{~nm} / 490 \mathrm{~nm}$ (mean \pm SEM, $\mathrm{n}=3$ ).

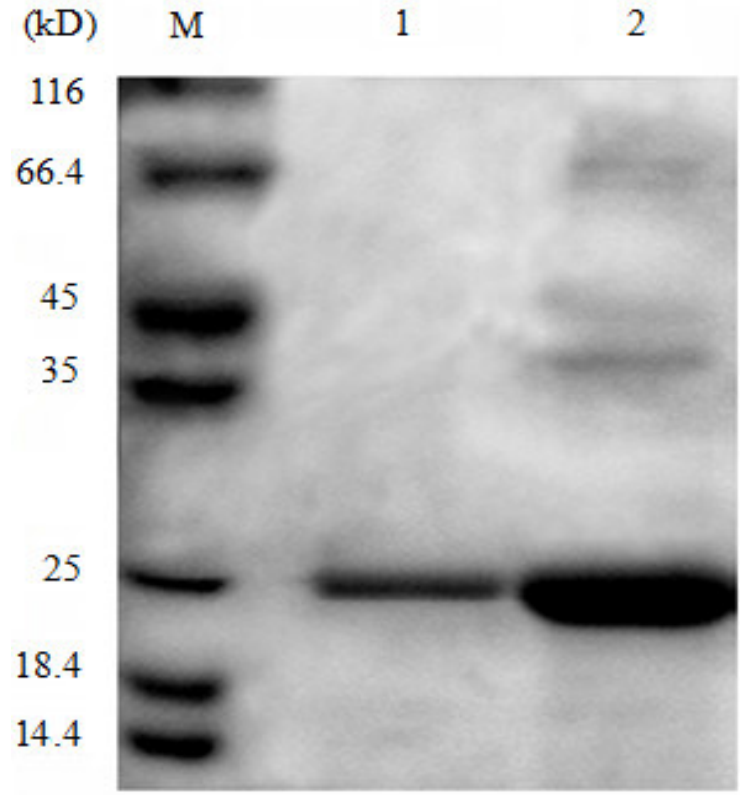

Figure 7. His pull-down assay. The purified $6 \times$ HisBmICAD and total extracted protein from Bm5 cells were loaded onto a HiTrap ${ }^{\mathrm{TM}}$ Desalting Column overnight. After washing, the bound proteins were separated by SDS-PAGE. Lane M, protein molecularweight marker (values are in $\mathrm{kDa}$ ); lane 1, the control group, eluted proteins after the incubation of $\mathrm{Bm} 5$ cell total extracted protein with HiTrap ${ }^{\mathrm{TM}}$ Desalting Column; lane 2, experimental group, eluted proteins after the incubation of total extracted protein from $\mathrm{Bm} 5$ cells with column purified $6 \times$ His-BmICAD. al., 1998; Lechardeur et al., 2000), but in the cytoplasm of activated thymocytes and Hodgkin's lymphoma cells (Nagata et al., 2002; Xerri et al., 2000). These contradictory results may indicate that ICAD is localized in the nucleus or cytoplasm in a cell-specific manner. Our subcellular localization results showed that BmICAD was mainly found in cytoplasm near the nucleus. This result is in agreement with previous results in Drosophila; Drosophila ICAD does not have a nuclear localization signal (NLS) at the position corresponding to the NLS of mammalian ICAD proteins (Yokoyama et al., 2000; Mukae et al., 2000). Therefore, insect ICAD may be also localized to the cytoplasm due to the lack of a NLS. The localization of BmICAD in cytoplasm is near to nucleus, it might facilitate the released CAD to enter the nucleus and thus, cause DNA cleavage and nuclear enrichment (Sakahira et al., 1998; Liu et al., 1998).

In the protein interaction studies, several specific bands were found, five different protein points were selected and peptide sequences were further determined by MS. According to the obtained PMF, we searched the NCBI protein data bank, only two possible interacting proteins, BmSCF and BmSPARC, were found. We couldn't find more information about the other three samples; they might be new proteins which are not included in the protein data bank. We will perform extensive studies to address this in our next work.

As well known, the primary role of BmSCF is as a DNA helicase (Ohta et al., 1995); it is necessary to uncoil the super-helix structure of DNA to facilitate the cleavage of 
Table 1. The identification result of possible BmICAD-binding proteins.

\begin{tabular}{lccl}
\hline Spot ID & Mr & pl & Protein name \\
\hline gil 112983659 & 37870.9 & 4.49 & DNA supercoiling factor (Bombyx mori) \\
gil 114051654 & 37359.2 & 4.86 & secreted protein acidic and rich in cysteine (Bombyx mori) \\
\hline
\end{tabular}

Spot ID is the accession number from NCBInr; Mr: molecular mass (kD); pl: isoelectric point.

DNA. BmSCF was thought to localize to the nucleus, but our results show that BmICAD was localized to the cytoplasm (Figure 3) raising the question of how the two proteins can interact. BmSPARC can inhibit cell proliferation and induce apoptosis in tumor cells (Tai and Tang, 2008). However, the mechanism by which BmICAD interacts with BmSCF to inhibit cell apoptosis and the effects of BmSPARC on BmICAD during silkworm cell apoptosis remains unknown. The answers to these questions will be important to understand the specific apoptotic mechanism during silkworm metamorphosis and the developmental phase.

\section{ACKNOWLEDGMENTS}

This work was supported by the National High-tech R \& D program (863 Program) (No.2011AA100603) and the Natural Science Foundation of Zhejiang Province (No.Y3090339).

\section{REFERENCES}

Dimmeler S, Haendeler J, Nehls M, Zeiher AM (1997). Suppression of apoptosis by nitric oxide via inhibition of interleukin-1beta-converting enzyme (ICE)-like and cysteine protease protein (CPP)-32-like proteases. J. Exp. Med., 185: 601-607.

Eckhart L, Fischer H, Tschachler E (2007). Phylogenomics of caspaseactivated DNA fragmentation factor. Biochem. Biophys. Res. Commun., 356: 293-299.

Enari M, Sakahira H, Yokoyama H, Okawa K, Iwamatsu A, Nagata S (1998). A caspase-activated DNase that degrades DNA during apoptosis, and its inhibitor ICAD. Nature, 391: 43-50.

Feng Q, Li P, Leung PC, Auersperg N (2004). Caspase-1, a new splice variant of the caspase-1 gene. Genomies, 84: 587-591.

Hoffmann R, Valencia A (2004). A gene network for navigating the literature. .Nat.. Genet. 36: p. 664.

Inohara N, Koseki T, Chen S, Wu X, Nunez G (1998). CIDE, a novel family of cell death activators with homology to the $45 \mathrm{kDa}$ subunit of the DNA fragmentation factor. EMBO J., 17: 2526-2533.

Kuroda K, Gröner A, Frese K, Drenckhahn D, Hauser C, Rott R, Doerfler W, Klenk HD (1989). Synthesis of biologically actived influenza virus haemagglutinin in insect larvae. J. Virol., 63: 16771685.

Lechardeur D, Drzymala L, Sharma M, Zylka D, Kinach R, Pacia J, Hicks C, Usmani N, Rommens JM, Lukacs GL (2000). Determinants of the nuclear localization of the heterodimeric DNA fragmentation factor (ICAD/CAD). J. Cell Biol., 150: 321-334.

Liu X, Zou H, Slaughter C, Wang X (1997). DFF, a heterodimeric protein that functions downstream of caspase-3 to trigger DNA fragmentation during apoptosis. Cell, 89: 175-184.

Liu X, Li P, Widlak P, Zou H, Luo X, Garrard WT, Wang X (1998). The $40-\mathrm{kDa}$ subunit of DNA fragmentation factor induces DNA fragmentation and chromatin condensation during apoptosis. Proc.
Natl. Acad. Sci. USA., 95: 8461-8466.

Mukae N, Yokoyama H, Yokokura T, Sakoyama Y, Sakahira H, Nagata S (2000). Identification and developmental expression of inhibitor of caspase-activated DNase (ICAD) in Drosophila melanogaster. J. Biol. Chem., 275: 21402-21408.

Nagata T, Kishi H, Liu QL, Matsuda T, Imanaka T, Tsukada K, Kang D, Muraguchi A (2002). The regulation of DNAse activities in subcellular compartments of activated thymocytes. Immunology, 105: 399-406.

Ohta T, Kobayashi M, Hirose S (1995). Cloning of a cDNA for DNA Supercoiling Factor Reveals Distinctive $\mathrm{Ca}^{2+}$-binding Protein. J. Biol. Chem., 270: 15571-15575.

Sakahira H, Enari M, Nagata S (1998). Cleavage of CAD inhibitor in CAD activation and DNA degradation during apoptosis. Nature, 391: 96-99.

Tai IT, Tang MJ (2008). SPARC in cancer biology: Its role in cancer progression and potential for therapy. Drug Resist. Updates, 11(6): 231-246.

Tettamanti G, Grimaldi A, Pennacchio F, de Eguileor M (2007). Lepidopteran larval midgut during prepupal instar: digestion or selfdigestion? Autophagy, 3(6): 630-631.

Tsuruta T, Oh-hashia K, Ueno Y, Kitade Y, Kiuchi K, Hirata Y (2007). RNAi knockdown of caspase-activated DNase inhibits rotenoneinduced DNA fragmentation in HeLa cells. Neurochem. Int., 50(4): 601-606.

Van de Craen M, Vandenabeele P, Declercq W, Van den Brande I, Van Loo G, Molemans F, Schotte P, Van Criekinge W, Beyaert R, Fiers W (1997). Characterization of seven murine caspase family members. FEBS Lett., 403: 61-69.

Wang ZX, Wang P, Yao L, Zeng ZD, Xu HF, Duan J, Wang GH, Xia QY (2006). Molecular cloning, sequence and function analyses and specific expression of gene BmICAD related to apoptosis in silkworm, Bombyx mori. Yi Chuan, 28(7): 838-844.

Wyllie AH (1980). Glucocorticoid-induced thymocyte apoptosisis associated with endogenous endonuclease activation. Nature, 284 (5756): 555-556.

Xerri L, Palmerini F, Devilard E, Defrance T, Bouabdallah R, Hassoun J, Birg F (2000). Frequent nuclear localization of ICAD and cytoplasmic co-expression of caspase-8 and caspase- 3 in human lymphomas. J. Pathol., 192: 194-202.

Yokoyama H, Mukae N, Sakahira H, Okawa K, Iwamatsu A, Nagata S (2000). A novel activation mechanism of caspase-activated DNase from Drosophila melanogaster. J. Biol. Chem., 275(17): 1297812986.

Yu W, Akishita M, Xi H, Nagai Ko, Sudoh No, Hasegawa H, Kozaki K, Toba K (2006). Angiotensin converting enzyme inhibitor attenuates oxidative stress-induced endothelial cell apoptosis via p38 MAP kinase inhibition. Clinica. Chimica. Acta, 364: 328-334. 